\title{
Heat transfer and pressure drop characteristics of gas-liquid Taylor flow in mini ducts of square and rectangular cross-sections
}

\author{
Jingzhi Zhang ${ }^{1,2}$, David F. Fletcher ${ }^{3}$, Wei Li ${ }^{1}$
}

1. Department of Energy Engineering, Zhejiang University, Hangzhou 310027, China

2. Department of Energy Engineering, Collaborative Innovation Center of Advanced Aero-Engine, Zhejiang University, Hangzhou 310027, China;

3. School of Chemical and Biomolecular Engineering, University of Sydney, Sydney, NSW2006, Australia

Corresponding author:

Name: Wei Li

Phone/Fax: +86057187952244

E-mail: weili96@zju.edu.cn 


\begin{abstract}
The thermal and flow characteristics of gas-liquid Taylor flow in vertical mini square and rectangular ducts were studied numerically for water and ethylene glycol as the liquid phase and nitrogen as the gas phase. The effects of fluid properties, flow parameters, and aspect ratio on the bubble shape, recirculation time, friction factor, and Nusselt number are discussed. The results indicate that the gas phase is confined by the tube wall in square and rectangular tubes leading to an asymmetrical Taylor bubble at low Capillary numbers, while an axisymmetric bubble is formed at high Capillary numbers. The liquid film thicknesses in the square and rectangular ducts are not uniform with a thicker liquid film formed at the tube corner. The recirculation region decreases and the dimensionless recirculation time increases with increasing Capillary number, which means that the intensity of recirculation decreases with increasing Capillary number. The friction factor decreases with increasing two-phase mixing velocity and aspect ratio and increases in gas void fraction, while the reverse is true for the two-phase Nusselt number. Compared with the ethylene glycol/nitrogen cases, the addition of Taylor bubble plays a more significant role on the pressure drop increase and heat transfer enhancement for the water/nitrogen cases because of the larger recirculation volume and smaller dimensionless recirculation time. Two empirical correlations are developed to predict the apparent slug Nusselt number and the film-to-slug Nusselt number for gas-liquid Taylor flow in square and rectangular ducts.
\end{abstract}

Keywords: Taylor flow; heat transfer; pressure drop; square ducts 


\section{NOMENCLATURE}

$A R \quad$ Aspect ratio, $h / w$

$c_{p} \quad$ Specific heat capacity, $\mathrm{J} / \mathrm{kg} \mathrm{K}$

$\mathrm{Ca} \quad$ Capillary number, $\mu_{L} v_{t p} / \sigma$

$d \quad$ Hydrodynamic diameter, $2 w h /(w+h)$

$h \quad$ Duct height, $\mathrm{m}$

$H \quad$ Enthalpy, $\mathrm{J} / \mathrm{kg}$

$f_{\mathrm{s}} \quad$ Friction factor in the liquid slug, $\Delta P d /\left(2 \rho_{L} v_{t p}{ }^{2} L_{s}\right)$

$\boldsymbol{F} \quad$ Surface tension force, $\mathrm{N} / \mathrm{m}^{3}$

g Gravity acceleration, $9.81 \mathrm{~m} / \mathrm{s}^{2}$

$k$ Thermal conductivity, $\mathrm{W} / \mathrm{m} \mathrm{K}$

$L_{c} \quad$ Length of the computational domain, $m$

$L_{s} \quad$ Length of the liquid slug, $\mathrm{m}$

$L_{s}{ }^{*} \quad$ Dimensionless slug length, $L_{s} /\left(\operatorname{Pr}_{L} \operatorname{Re}_{t p} d\right)$

$\mathrm{Nu} \quad$ Nusselt number

$\mathrm{Nu}_{l o} \quad$ Nusselt number for the fully-developed single-phase flow

$P \quad$ Pressure, $\mathrm{Pa}$

$q_{w} \quad$ Wall heat flux, $\mathrm{W} / \mathrm{m}^{2}$

$Q \quad$ Flow rate, $\mathrm{m}^{3} / \mathrm{s}$

Re Two-Phase Reynolds number, $v_{t p} \rho_{L} d / \mu_{L}$

$t \quad$ Time, $\mathrm{s}$

$T \quad$ Temperature, $\mathrm{K}$

$\boldsymbol{v} \quad$ Velocity, $\mathrm{m} / \mathrm{s}$

$v_{r e l} \quad$ Slip velocity, $1-v_{t p} / v_{b}$

$V \quad$ Volume, $\mathrm{m}^{3}$

w Duct width, $\mathrm{m}$

\section{Greek letters}

$\alpha \quad$ Volume fraction

$\kappa \quad$ Interface curvature, $1 / \mathrm{m}$

$\mu \quad$ Dynamic viscosity, $\mathrm{N} / \mathrm{m}$ 
$\varepsilon \quad$ Gas volume fraction in a unit cell

$\rho \quad$ Density, $\mathrm{kg} / \mathrm{m}^{3}$

$\sigma \quad$ Surface tension coefficient, N/m

$\tau \quad$ Dimensionless recirculation time

\section{Subscripts}

$b$, in Liquid bulk value for velocity inlet

$b$,out Liquid bulk value for pressure outlet

corner Tube corner

G $\quad$ Gas phase

$L \quad$ Liquid phase

tp Two phase, average value for a computational domain

w Wall 


\section{Introduction}

With the development of microreactors, compact heat exchangers, and micro heat sinks, more attention has been paid to the heat and mass transfer and frictional characteristics in mini/micro channels. The heat and mass transfer characteristics of mini/micro channels are similar to those of conventional ones for single-phase flow, while the surface tension effect gradually dominates the twophase flow with decreasing channel hydraulic diameter. Compared with single-phase flow, the twophase flow has more potential to reach the increasing demand for high-flux thermal management solutions by taking advantage of the latent heat in phase-change flows like condensation and boiling, or internal recirculations for gas-liquid and liquid-liquid Taylor flow. Taylor flow is more stable and controllable than flows with phase change and has a relatively higher heat transfer coefficient compared with single-phase flow.

Numerous experimental studies have been conducted on the flow and thermal characteristics of Taylor flow in circular and square channels in terms of Taylor bubble shapes [1-2], bubble velocities [3], slug lengths [1, 4], liquid film thickness [5-6], flow fields [7-8], pressure drops [9-10], and heat transfer characteristics [1, 11-14]. Exhaustive reviews about these have been performed by Angeli and Gavriilidis [15], Gupta et al. [16], Eain et al. [17], and Bandara et al. [18].

Taylor flow is characterized by periodic capsular bubbles separated by liquid slugs with bubble and slug lengths larger than the channel hydraulic diameter [1]. Akbar and Ghiaasiaan [19], Laborie et al. [20], and Liu et al. [21] developed various correlations, which included the gas and liquid superficial velocities and properties, to predict the liquid and bubble lengths. However, the inlet distribution also plays a significant role on the liquid and gas lengths as shown in Leung et al. [12] and Shao et al. [22]. Though Kreutzer et al. [23] developed a correlation to predict slug lengths in multiphase monoliths using measured pressure drops, this method only showed good prediction for short slugs (shorter than seven times of the channel diameter) for which the pressure drop was sensitive to slug length variations.

A thin liquid film is formed between the Taylor bubble and the channel wall, which plays an important role in heat and mass transfer $[1,11]$. The liquid film thickness is mainly determined by the Capillary number $(\mathrm{Ca})$ as indicated in the correlations developed for circular channels by Irandoust and Andersson [24], Aussillous and Quéré [25], and Han and Shikazono [5]. However, Taylor bubbles 
are confined by the tube wall in square and rectangular ducts, and a thicker liquid film is formed at the corners [26]. Thulasidas et al. [27-28] studied Taylor flow in square channels and pointed out that the transition from a non-axisymmetric to an axisymmetric bubble occurs at $C a \approx 0.04$.

A recirculating flow, which could efficiently enhance the heat and mass transfer in the continuous phase, is induced by two-phase interfaces. The visualization of this recirculating flow has been conducted by Devoria et al. [7] and Meyer et al. [8] using micro-PIV technology. The pressure drop also increases with the addition of a second incompressible phase. Kreutzer et al. [10], Abiev [29], and Warnier et al. [9] proposed empirical models for the frictional pressure drops of Taylor flow. However, these correlations assumed an axisymmetric Taylor bubble with a uniform thickness liquid film. Compared with the flow characteristics, limited work has been done on the heat transfer characteristics of Taylor flow. Though the experimental studies in circular tubes [1, 11-12, 14] and square and rectangular channels [30] all show significant enhancement of heat transfer compared with their singlephase counterparts, the values of this enhancement varied in different studies. As pointed out in Bandara et al. [18], there was little agreement in the heat transfer coefficient correlations proposed by Kreutzer et al. [31], Walsh et al. [14] and Leung et al. [1, 12].

Numerical simulation can serve as a complementary method to investigate the flow and heat and mass transfer characteristics of Taylor flow with less expense. Most of the numerical studies about Taylor flow are performed in two-dimensional channels using a fixed reference frame [32-34] or a moving frame [35-38]. A brief review of the numerical work on Taylor flow has been conducted by Talimi et al [39]. According to Gupta et al. [34], the near wall mesh must be sufficiently fine, at least five mesh elements across the film, to capture the thin liquid film present in Taylor flow. However, the two-dimensional simulation cannot consider the flow and heat and mass transfer characteristics in channels with non-circular cross-sections. For the three-dimensional numerical work, the moving frames reference is mostly adopted to save the computational time. Taha and Cui [40-41] studied the bubble shapes and streamlines of gas-liquid Taylor flow in square channels. Liu and Wang [21] investigated the hydrodynamics of Taylor flow in square and triangular capillaries. Falconi et al. [42] studied numerically and experimentally local flow phenomena in co-current upward laminar Taylor flow through a small square channel. A local backflow region in the rear part of the liquid film was observed. Abadie et al. [43] analyzed the mixing and recirculation characteristics of gas-liquid Taylor flow in square and rectangular ducts. The numerical results indicate that the recirculation volumes 
decrease and the recirculation times increase with increasing aspect ratio. Abiev [44] pointed out that recirculation times were depended on slug lengths and that circulation occurred even in very short liquid slugs (about 0.17 times of the tube diameter). The three-dimensional numerical work about heat transfer characteristics in non-circular channels is limited. Talimi et al. [45] studied numerically the heat transfer characteristics of Taylor flow in square microchannels under constant heat flux boundaries. The liquid film and the gas phase were neglected, and the bubble shapes were determined by the contact angles. However, the liquid films at the duct corners exist even at lower Capillary number for the case of square channels. As a consequence, the numerical Nusselt number was higher than that of the experimental work of Betz and Attinger [30]. As stated in Dai et al. [11], the thermal resistance contribution of the liquid film is important for the heat transfer performance of Taylor flow. Neglecting this part will lead to a higher heat transfer enhancement compared with the single-phase flow especially at higher $\mathrm{Ca}$. Che et al. [46] conducted three-dimensional simulations in square and rectangular channels under constant temperature boundary conditions taking the thin liquid film into account. The effects of droplet length, aspect ratio, and Péclet number on the heat transfer process in liquid-liquid Taylor flow were analyzed.

Though numerous works has been published, the characteristics of the fully-developed gas-liquid Taylor flow in square and rectangular channels have not been investigated in detail. With the purpose of bridging this research gap, the present numerical study is designed to provide further understanding of the flow and thermal characteristics of Taylor flow in square and rectangular ducts under constant heat flux boundary conditions. Water (W) and ethylene glycol (EG) are used as the liquid phase, covering a wide range of the Capillary numbers $(0.0011 \leq C a \leq 0.190)$.

\section{Numerical model}

A commercial CFD code (ANSYS Fluent, Release 14.0) which is based on the finite volume method is used to model the gas-liquid Taylor flow in mini ducts. The gas-liquid interface is tracked using the volume of fluid (VOF) method, in which the gas and liquid phases are assumed incompressible and do not penetrate each other. The interface is captured by solving an additional advection equation for the volume fraction. A single-fluid (homogeneous) formulation is applied throughout the computational domain where velocity and pressure fields are shared amongst the two phases. Properties of the single-fluid are calculated based on the volume weighted fraction of each 
phase in the cell.

\subsection{Governing equations}

As discussed in Asadolahi et al. [38], fully-developed Taylor flow is an unsteady, periodic flow in the laboratory frame of reference, but becomes steady in a reference frame moving with the velocity of the disperse phase. Two different methods are able to model the reference frame motion: dynamic mesh method (DMM) and frame motion method (FMM). A detailed description of the DMM method is presented in Asadolahi et al. [38]. As the bubble velocity ( $\left.\boldsymbol{v}_{\mathbf{b}}\right)$ is not known, it is calculated at the beginning of each time step using the volume-fraction-weighted gas axial velocity:

$$
v_{b}=\frac{\int \alpha_{G} v_{z} d V}{\int \alpha_{G} d V}
$$

For the FMM, the absolute velocity formulation is used, and the governing equations are shown below: Continuity Equation:

$$
\frac{\partial \rho}{\partial t}+\nabla \cdot\left[\rho\left(v-v_{b}\right)\right]=0
$$

Momentum Equation:

$$
\frac{\partial\left[\rho\left(\boldsymbol{v}-\boldsymbol{v}_{\boldsymbol{b}}\right)\right]}{\partial t}+\nabla \cdot\left[\rho \boldsymbol{v}\left(\boldsymbol{v}-\boldsymbol{v}_{\boldsymbol{b}}\right)\right]=-\nabla P+\nabla \cdot\left[\mu\left(\nabla \boldsymbol{v}+\nabla \boldsymbol{v}^{T}\right)\right]+\rho \boldsymbol{g}+\boldsymbol{F}
$$

Energy Equation:

$$
\frac{\partial(\rho H)}{\partial t}+\nabla \cdot\left[\rho\left(\boldsymbol{v}-\boldsymbol{v}_{\boldsymbol{b}}\right) H\right]=\nabla \cdot[k(\nabla T)]
$$

Volume Fraction Equation:

where

$$
\begin{gathered}
\frac{\partial(\alpha)}{\partial t}+\left(\boldsymbol{v}-\boldsymbol{v}_{\boldsymbol{b}}\right) \cdot \nabla \alpha=0 \\
\rho=\alpha_{L} \rho_{L}+\alpha_{G} \rho_{G} \\
\mu=\alpha_{L} \mu_{L}+\alpha_{G} \mu_{G} \\
k=\alpha_{L} k_{L}+\alpha_{G} k_{G} \\
\alpha_{L}+\alpha_{G}=1
\end{gathered}
$$


The continuum surface force (CSF) model, proposed by Brackbill et al. [47], is adopted in this work. In this model, interfaces between different fluids are treated as transition zones with finite thickness. The surface tension effect is included in the source term $\boldsymbol{F}$ in eq. (3), which is defined as follows:

$$
\boldsymbol{F}=\sigma \frac{\rho}{0.5\left(\rho_{l}+\rho_{v}\right)} \kappa \nabla \alpha_{l}
$$

where $\sigma$ is the interfacial tension force between liquid and gas. The gradient of the volume fraction scalar is adopted to calculate the interface curvature, $\kappa$, via

$$
\kappa=\nabla \cdot \frac{\nabla \alpha_{L}}{\left|\nabla \alpha_{L}\right|}
$$

\subsection{Computational domain and mesh independence}

In the present work, the width $(w)$ of the square duct is $1 \mathrm{~mm}$, while the height $(h)$ varies from 1 to $2 \mathrm{~mm}$, corresponding to the aspect ratio $(A R)$ ranging from 1 to 2 . The length of the computational domain which contains a Taylor bubble and two halves of adjacent liquid slugs is $8 \mathrm{~mm}$, i.e. the unit cell length is maintained constant in this work. Fig. 1 shows the geometry of the computational domain, boundary conditions, and a cross-section of the computational mesh. As the flow is symmetric, only a quarter of the square domain is simulated with two symmetry planes in the $x z$ and $y z$ directions. The no-slip boundary condition and a constant wall heat flux of $32 \mathrm{~kW} / \mathrm{m}^{2}$ are employed at the upper and right-hand walls. The velocity inlet and pressure outlet boundary conditions are applied at the upstream and downstream boundaries, respectively. The properties of nitrogen $\left(\mathrm{N}_{2}\right)$, water $(\mathrm{W})$, and ethylene glycol (EG) are listed in Table. 1.

As recommended by Gupta et al. [34], a fine near-wall mesh having at least five elements, is necessary for capturing the thin liquid film in Taylor flow. In the present work, the meshes near the upper and right walls are refined to capture the thin liquid film. The gas-liquid interface profiles obtained using three different grid sizes are shown in Fig. 2. Bubble shapes and liquid film thickness are nearly identical for the grid density with more than $40 \times 40 \times 320$. The mesh density of $40 \times 40 \times 320$ is adopted for the simulations for the square duct. The number of mesh elements in the $y$ direction are scaled by $A R$ for rectangular domains, while the mesh distributions remain the same along the $x$ and $z$ direction. 


\subsection{Numerical method and solver options}

Before the two-phase flow simulation is started, a steady periodic single-phase flow simulation is conducted to obtain the fully-developed flow field for the continuous phase. The computational domain and mesh distribution for the single-phase work are the same as those in the two-phase simulation. Periodic boundary conditions are employed at the inlet and outlet of the ducts. After obtaining the fully-developed flow field for the continuous phase, the velocity components are interpolated onto the two-phase numerical domain as the initial velocities for the liquid and gas phases.

The same method as that used by Asadolahi et al. [38] is adopted for the simulations of squareand rectangular-section ducts. Initially, a rectangular bubble is set in the middle of the computational domain with the volume equal to the product of the gas hold-up and the unit cell volume. The velocity components at the inlet boundary are set to the values at the downstream pressure outlet. When the velocity of the Taylor bubble and the pressure at the velocity inlet are stable, the flow field is frozen, and the energy equation is solved. The scaled temperature profile is wrapped from faces where flow leaves the domain to the corresponding ones at the opposite end of the unit cell, as depicted in Fig. 1. The temperature difference due to heat addition at the wall is calculated via eq. (12) and is used to adjust the inlet temperature derived from the outlet temperature. A more detailed description of this method and the derivation of eq. (12) can be found in Asadolahi et al. [38].

$$
\Delta T_{C}=\frac{4 q_{w} L_{c} / d}{\left[\left(v_{t p}-\varepsilon_{G} v_{b}\right) \rho_{L} c_{p L}+\varepsilon_{G} v_{b} \rho_{G} c_{p G}\right]}
$$

The iterative fractional step scheme is used for the time-marching of the continuity, momentum, energy, and volume fraction equations. The implicit body force treatment is adopted to take the body forces into account. For the momentum and energy discretization, the QUICK scheme is employed. The body-force-weighted and piecewise-linear interface calculation scheme are used for the discretization of pressure and volume fraction, respectively. A variable time step, controlled by a specified maximum value of Courant number, is used for the momentum and pressure equations, while a constant time-step of $0.1 \mathrm{~ms}$ is applied for the energy equation.

\section{Results and discussion}

26 cases, covering a wide range of $R e(4.87 \leq R e \leq 500)$ and $C a(0.0011 \leq C a \leq 0.190)$, were 
performed to investigate the hydrodynamics and heat transfer characteristics of gas-liquid Taylor flow in square and rectangular ducts. The flow conditions, working fluids and non-dimensional numbers considered are shown in Table 2.

\subsection{Comparison of the dynamic mesh method and the frame motion method}

Fig. 3 shows the axial velocity contours obtained using the DMM and FMM, respectively. The results are almost identical for these two methods. Compared with the DMM, the FMM is computationally cheaper. For the same case conducted using the same workstation with 8 processors and 16 GB RAM, it took about 17.4 hours and 24 hours to obtain the fully-developed Taylor flow using FMM and DMM, respectively. It should be noted that the UDFs for obtaining bubble velocities and wrapping boundary conditions are parallelized to fully utilize the workstation. For the DMM, it takes some time to update the computational mesh at the beginning of the time-step, which increases the computational time. In the following work, the FMM method is adopted.

\subsection{Bubble shapes, bubble lengths, and liquid film thickness}

Fig. 4 shows the shapes of the Taylor bubble in square- and rectangular-section ducts for different two-phase inlet velocities, gas void fractions, and aspect ratios. The Taylor bubble is confined by the duct wall at lower $C a(0.0011 \leq C a \leq 0.0055)$ for the $\mathrm{W} / \mathrm{N}_{2}$ case, leading to an asymmetrical bubble shape, while a symmetrical Taylor bubble is formed for the EG/N $\mathrm{N}_{2}$ cases with higher $C a(0.038 \leq C a$ $\leq$ 0.190). Compared with the bubble nose, a flatter bubble tail is obtained in vertical square- and rectangular-section ducts. As the Taylor bubble is confined in the radial direction, it mainly develops along the axial direction in the upstream and downstream directions. The development direction at the bubble tail is opposite to the flow direction, thus the development is hindered by the up-flowing fluid, leading to a flatter bubble tail.

With increasing two-phase mixing velocity, the bubble nose becomes sharper, while the bubble tail becomes flatter. A bullet-like Taylor bubble is observed at $C a \geq 0.038$ for the $\mathrm{EG} / \mathrm{N}_{2}$ cases. Compared with the W/ $\mathrm{N}_{2}$ cases, the surface tension effect is less significant for $\mathrm{EG} / \mathrm{N}_{2}$ cases in which the viscous forces dominate the Taylor flow. Araújo et al. [37] stated that the convex shape of the bubble tail flattens as the dominance of surface tension decreases and a concave shape is formed at 
higher Eötvös number. Moreover, the curvatures of the bubble nose and tail are more sensitive to the influence of mixture velocity for the EG/ $\mathrm{N}_{2}$ cases. Some ripples are obtained near the bubble tail at the duct corner for the $\mathrm{W} / \mathrm{N}_{2}$ cases, while these ripples are not observed for the $\mathrm{EG} / \mathrm{N}_{2}$ cases. Leung et al [1] reported similar experimental results for Taylor flow in a $2 \mathrm{~mm}$ vertical circular tube using water, $50 \mathrm{wt} \%$ ethylene glycol/ water mixture, and pure ethylene glycol as the continuous phases.

The bubble length increases with increasing gas void fraction, corresponding to a decrease in liquid slug length for a fixed unit cell length. The shapes of the bubble tail and nose are not influenced by the gas void fraction, while the length of the constant liquid film region decreases with decreasing gas void fraction. At lower gas void fraction, the constant liquid film region eventually disappears. With increasing $A R$, the height of the rectangular duct increases for a fixed duct width and the Taylor bubble is confined in the width (shorter edge) direction, while a thicker liquid film is formed in the height (longer edge) direction. The uneven liquid film thickness in the two directions will influence the mass and heat transfer performance at the side walls in rectangular ducts.

Detailed comparisons of Taylor bubble shapes and gas slug lengths obtained at various two-phase inlet velocities, gas void fractions, and aspect ratios are shown in Fig. 5. As shown in Fig. 5 (a), the bubble length increases with increasing two-phase mixing velocity at a fixed gas void fraction and unit cell length. At the same void fraction and two-phase mixing velocity, a longer Taylor bubble with a thicker liquid film is formed for the EG/N $\mathrm{N}_{2}$ case compared with the $\mathrm{W} / \mathrm{N}_{2}$ case. According to Abiev [48], the product of the bubble length and the mean bubble cross-sectional area is constant for a fixed unit cell with the same gas void fraction. The cross-sectional area of a bubble decreases with increasing velocity and increasing Capillary number because of the liquid film thickening [25, 49], leading to a longer bubble length at a higher velocity and Capillary number. The curvature of the bubble nose increases with increasing two-phase mixing velocity, while that of the bubble tail decreases with increasing two-phase mixing velocity. The liquid film thickness for the $\mathrm{W} / \mathrm{N}_{2}$ case is smaller than the $\mathrm{EG} / \mathrm{N}_{2}$ case at the same two-phase mixing velocity because of the lower $\mathrm{Ca}$. The gas void fraction has only a slight effect on the liquid film thickness and the bubble tail and nose curvatures, while a longer Taylor bubble is expected at higher gas void fraction.

Fig. 5 (b) shows the effect of $A R$ on Taylor bubbles at the same $C a$ and gas void fraction. The bubble shapes are scaled by the width of the square and rectangular ducts. As shown in Fig. 5 (b), the bubble length increases with increasing $A R$. In the width direction, the bubbles are confined by the 
side walls and the effect of $A R$ on the liquid film thickness is insignificant. The bubbles are not confined in the height direction, and the curvatures of the Taylor bubble tail and nose in the height direction decrease with increasing $A R$. The length of the constant liquid film region in the height direction decreases with increasing $A R$, and eventually disappears at higher $A R$. Compared with the $\mathrm{W} / \mathrm{N}_{2}$ cases, the effect of $A R$ on the length of the constant liquid film region is lower for the $\mathrm{EG} / \mathrm{N}_{2}$ cases because of the reduced effect of surface tension.

The cross-sectional shapes of Taylor bubble at the centre of square ducts, where a constant liquid film is formed, are shown in Fig. 6. As mentioned before, the Taylor bubble is confined by the tube wall, leading to an asymmetrical bubble shape at lower $C a$ as shown in Fig. 6 (a). The liquid film thickness at the duct corner is much higher than that at the centre of the duct wall. Kolb and Cerro [50] concluded that the corners are affected by larger viscous forces than the regions near the centre of the walls resulting in the asymmetrical bubble shapes. With increasing $C a$, the liquid film thickness at the duct corner increases and that at the centre of the duct wall remains nearly constant, thus decreasing the radius difference for the Taylor bubble. At higher $C a$, the Taylor bubble gradually becomes symmetric, with the liquid film thicknesses at the wall centre and the duct corner increase with increasing $\mathrm{Ca}$. Thulasidas et al. [27] stated that the transitional value of $\mathrm{Ca}$, from asymmetrical to symmetrical bubble shapes in square tubes is 0.04 . In the present work, a symmetrical Taylor bubble is observed at $C a=0.038$. It should be noted that the transitional $C a$ suggested in Thulasidas et al. [27] is based on the bubble velocity whereas here the two-phase mixing velocity is used in the present work.

Kreutzer et al. [51] analysed the liquid film thickness at the duct corner and proposed a correlation to predict it based on the experimental data of Kolb and Cerro [50], Thulasidas et al. [27], and the numerical data of Hazel and Heil [52]. As shown in eq. (13), the dimensionless liquid film thickness is mainly determined by $C a$. When $C a \rightarrow 0$, a constant value is obtained, which means that a liquid film still exists at the tube corner even at low $\mathrm{Ca}$. Han and Shikazono [6] measured the liquid film thickness in three square ducts with hydraulic diameter ranging from 0.3 to $1 \mathrm{~mm}$ using ethanol, water, and FC-40 as the liquid phase. A correlation for the liquid film thickness at the corner was proposed, in which the effect of inertia is considered by adopting We in this correlation as shown in eq. (14). Comparisons of the numerical data with the predicted values based on these two correlations are shown in Fig. 6 (b). The numerical data fit well with the two correlations at lower $C a$ for the W/ $\mathrm{N}_{2}$ cases, while both correlations underestimate the liquid film thickness at higher $C a$ for the EG/N 2 cases. The 
highest relative error is around $10 \%$ for these correlations.

$$
\begin{gathered}
\frac{2 \delta_{\text {corner }}}{d}=0.714+0.5 \exp \left(-2.25 C a^{0.445}\right) \\
\frac{2 \delta_{\text {corner }}}{d}=\frac{2.43 C a^{2 / 3}}{1+7.28 C a^{2 / 3}-0.255 W e^{0.215}}-0.243
\end{gathered}
$$

\subsection{Streamlines and recirculation times}

Fig. 7 shows the streamlines on a diagonal plane, bubble shape, and recirculation volumes at $\mathrm{Ca}$ $=0.114, \varepsilon_{G}=0.3$, and $A R=1$. The recirculation volumes in the liquid slug region at the front of the bubble nose are unshaded to give a better presentation of the streamlines there. As shown in Fig.7 (a), two recirculations, which can enhance the heat and mass transfer in Taylor flow, form in the liquid and gas slug in the frame of reference attached to the Taylor bubble. Gupta et al. [35] pointed out that the recirculation in the gas slug has only a very slight contribution to the heat transfer because of the relatively low thermal mass of the gas, while it will enhance the heat transfer in liquid-liquid Taylor flow. The streamlines at the tube corners are parallel to the flow direction. As recommended by Abadie et al. [43], the flow rate in the liquid slug can be divided into three parts: the volume $\left(V_{0}\right)$ of a positive flow rate $\left(Q_{0}\right)$; the volume $\left(V_{1}\right)$ of a negative flow rate $\left(Q_{1}\right)$, where $Q_{1}+Q_{0}=0$; the remainder of the volume $\left(V_{2}\right)$. The transition surfaces for these three regions are illustrated in Fig.7 (a).

Fig.7 (b) shows the cross-sectional shapes of the recirculation regions in square tubes at $\mathrm{Ca}=$ $0.0033,0.0055,0.114$, and 0.190 . The shapes of the recirculation regions are nearly identical at lower $\mathrm{Ca}$, while they shrink towards the centre of the tube and become circular in shape at higher $\mathrm{Ca}$. The recirculation region decreases in size with increasing $\mathrm{Ca}$ and eventually disappears when the bubble velocity is greater than the maximum liquid velocity. This will influence the heat and mass transfer in Taylor flows. The liquid film thickness of $V_{2}$ shown in Fig. 7 (b) is different from the liquid film thickness around the bubble as shown in Fig. 6 (a). Abiev [44] pointed out that at very low slug length, the bypass flux is greater than the direct flux $Q_{0}$. The intensity of recirculation in Taylor flow is quantified using the characteristic time $\tau_{1}$ which is defined as the time required for a fluid element to complete one circulation in the recirculation slug and $\tau_{2}$ which is defined as the time required for a fluid element to move from one end of the slug to the other end [53]. Thulasidas et al. [27] defined a dimensionless recirculation time $\tau_{\mathrm{rc}}$ as the ratio of $\tau_{1}$ and $L_{\mathrm{s}} / v_{\mathrm{b}}$ and $\tau_{0}$ as the ratio of $\tau_{2}$ and $L_{\mathrm{s}} / v_{\mathrm{b}}$. Fig. 7 
(c) depicts the effect of slip velocity, $v_{r e l}=1-v_{\mathrm{tp}} / v_{\mathrm{b}}$, on the dimensionless recirculation time $\tau_{0}$ and $\tau_{\mathrm{rc}}$ defined in eq. (15) and eq. (16), respectively. As shown in Fig. 7 (c), $\tau_{0}$ and $\tau_{\text {rc }}$ increase with increasing slip velocity, and the present numerical work fits well with that of Abadie et al. [43].

$$
\begin{gathered}
\tau_{0}=\frac{V_{0} / Q_{0}}{L_{s} / v_{b}} \\
\tau_{r c}=\frac{\left(V_{0}+V_{1}\right) / Q_{0}}{L_{s} / v_{b}}
\end{gathered}
$$

\subsection{Frictional characteristics}

The frictional behaviour of Taylor flow is of importance as it affects the design, stability, and energy loss of the system. Fig. 8 shows the effects of $v_{\mathrm{tp}}, \varepsilon_{G}$, and $A R$ on the liquid slug friction factor $f_{s}$, defined in eq. (17) in which the frictional pressure drop is obtained by subtracting the gravitational pressure drop from the total pressure drop. The friction factors for the fully-developed single-phase flow in square and rectangular ducts are also presented in Fig. 8. These friction factors are obtained from Bahrami et al. [54], in which pressure drops of the fully-developed laminar flow in microchannels with arbitrary cross-section were studied.

$$
f_{s}=\frac{\Delta P d}{2 L_{s} \rho_{L} V_{t p}^{2}}
$$

As shown in Fig. 8, $f_{\mathrm{s}}$ is higher than that of the fully-developed single-phase flow because of the addition of a second immiscible phase to the flow. The presence of Taylor bubbles disturbs the flow field of the liquid slug near the bubble and increases the pressure drop in the liquid phase. From Fig. 8, one can also conclude that $f_{\mathrm{s}}$ decreases with increasing $v_{t p}$ and $A R$ and with decreasing $\varepsilon_{G}$. A higher $f_{\mathrm{s}}$ is obtained for the $\mathrm{EG} / \mathrm{N}_{2}$ case at the same condition because of the higher viscosity of the liquid phase. Compared with the EG/ $\mathrm{N}_{2}$ cases, the addition of Taylor bubbles plays a more significant role on the pressure drop increase for the $\mathrm{W} / \mathrm{N}_{2}$ cases.

Kreutzer et al. [10] studied Taylor flow in circular tubes numerically and experimentally and proposed a correlation, eq. (18), to predict the pressure gradients of the gas-liquid Taylor flow. As shown in eq. (18), for an infinitely long liquid slug, the pressure gradient for Taylor flow tends to that for single-phase flow. For square and rectangular ducts, the $\left(f R e_{\mathrm{tp}}\right)_{\mathrm{lo}}$, obtained from Bahrami et al [54], is lower than that of circular tubes which is 16. Fig. 9 shows the comparison of the simulated pressure 
gradients and those calculated using eq. (18). As shown in Fig. 9, eq. (18) tends to underestimate the pressure gradients for the $\mathrm{W} / \mathrm{N}_{2}$ cases, while it fits well for the EG/ $\mathrm{N}_{2}$ cases. Overall, the correlation can predict the numerical data well with all data falling inside the $\pm 20 \%$ error band for both cases. The mean absolute deviation (MAD), defined in Eq. 19, is 6.3\%.

$$
\begin{gathered}
(-d P / d z)=\frac{2\left(f R e_{t p}\right)_{l o}}{\operatorname{Re}_{t p}}\left[1+0.07 \frac{d_{h}}{L_{s}}\left(\frac{R e}{C a}\right)^{0.33}\right] \frac{\rho_{L} v_{t p}^{2}}{d_{h}} \frac{L_{s}}{L_{c}} \\
M A D=\frac{1}{n} \sum_{1}^{n}\left|\frac{U_{p r e}-U_{s i m}}{U_{s i m}}\right| \bullet 100 \%
\end{gathered}
$$

where $U$ is the value of a numerical or calculated data.

\subsection{Heat transfer characteristics}

An average Nusselt number $N u_{t p}$ calculated using eq. (20) is adopted to investigate the heat transfer characteristics of the gas-liquid Taylor flow,

$$
N u_{t p}=\ln \left(\frac{T_{w}-T_{b, \text { in }}}{T_{w}-T_{b, \text { out }}}\right) \frac{q_{w}}{T_{b, \text { out }}-T_{b, \text { in }}} \frac{d}{k_{L}}
$$

where $T_{b, \text { in }}$ and $T_{b, \text { out }}$ are the enthalpy flux-weighted average fluid temperature at the velocity inlet and pressure outlet, while $T_{w}$ is obtained using the area-weighted average temperature at the duct wall.

The effects of $v_{t p}, \varepsilon_{G}$, and $A R$ on $N u_{t p}$ are shown in Fig. 10 together with the $N u_{l o}$ for the fullydeveloped single-phase flow in square- and rectangular-section ducts, obtained in the classical work of Shah and London [55]. The average Nusselt numbers for Taylor flow in square tubes is about 1.664 times that for the fully-developed single phase flow. $N u_{t p}$ increases with increasing $v_{t p}$ and $A R$ and decreases with increasing $\varepsilon_{G}$. Similar to the pressure drop characteristics, the additional second phase has more pronounced effect on the heat transfer enhancement for the $\mathrm{W} / \mathrm{N}_{2}$ cases than the $\mathrm{EG} / \mathrm{N}_{2}$ cases. The $N u_{t p}$ difference of these two cases at the conditions increases with increasing $v_{t p}$ and with decreasing $\varepsilon_{G}$.

Generally speaking, the advection effect is enhanced with increasing $v_{t p}$, thus increasing the heat transfer coefficient. However, as shown in Fig. 5 and Fig. 6, the liquid film thickness also increases with increasing $v_{t p}$, which will increase the thermal resistance in the liquid film and inhibit the heat 
transfer process. In the present work, the effect of enhanced advection is more pronounced than that of increasing thermal resistance in the liquid film, and $N u_{t p}$ increases with increasing $v_{t p}$. As stated in Gupta et al. [33], the contribution of the gas phase to heat transfer is negligible compared with the liquid phase. With increasing $\varepsilon_{G}$, the proportion of the liquid phase decreases, leading to a lower $N u_{t p}$. However, this is not so for the case of the liquid-liquid Taylor flow in which the disperse phase contributes significantly to the overall heat transfer. Dai et al. [11] and Che et al. [46] both reported that the Nusselt number increases with the homogenous void fraction of the disperse droplet because of the thermophysical properties of the disperse phase. With increasing $A R$, the heat transfer is enhanced at the side wall with the longer edge, while it is inhibited at the duct wall with the shorter edge [46]. As the side wall contributes more to the average heat transfer coefficient because of the higher surface area, the $N u_{t p}$ increases with increasing $A R$. However, the constant liquid film region decreases with increasing $A R$, leading to a decrease in the heat transfer enhancement at the side wall with the longer edge. As a consequence, $N u_{t}$ does not change much at higher $A R$.

Leung et al. [12] studied the heat transfer characteristics of Water/Nitrogen Taylor flow in a vertical circular tube experimentally and adopted an apparent Nusselt number $N u_{l}{ }^{*}$, defined in eq. (21), to characterize the liquid slug heat transfer. The heat transfer process in the liquid slug was analogous to the developing laminar flow, and $\mathrm{Nu}_{l}{ }^{*}$ was correlated using an inverse Graetz number for the liquid slug $L_{s}{ }^{*}$, eq. (24). However, this formulation, eq. (22), failed to predict the heat transfer characteristics for more viscous fluid in their following work [1] in which another formulation, eq. (23), for $N u_{l}{ }^{*}$ was proposed taking the effect of $\mathrm{Ca}$ into account.

$$
\begin{gathered}
N u_{l}^{*}=N u_{t p} L_{c} / L_{s} \\
N u_{l}^{*}=N u_{l o}+\frac{a_{1}}{L_{s}^{*}+a_{2} L_{s}^{* 1 / 3}} \\
N u_{l}^{*}=N u_{l o}+a_{1}\left(L_{s}^{*}\right)^{a_{2}}(C a)^{a_{3}} \\
L_{s}^{*}=L_{s} /\left(\operatorname{Re}_{t p} \operatorname{Pr}_{L} d\right)
\end{gathered}
$$

where

Recently, Dai et al. [11] divided the thermal resistance of the Taylor flow into three parts: the wall film resistance, the film-to-slug resistance, and the film-to-droplet resistance. The contribution of the wall film resistance increases with increasing $C a$ corresponding to the increase in the liquid film thickness, which is the reason for the failure of the previous formulation, eq. (22), of Leung et al. [12] to more 
viscous fluids. For the gas-liquid Taylor flow, the contribution of the gas phase to the overall heat transfer is negligible and the film-to-slug Nusselt number $N u_{f s}$ is defined as:

$$
N u_{f s}=\frac{L_{c}}{L_{s}\left(1 / N u_{t p}-\delta / d\right)}
$$

where the correlation proposed by Aussillous and Quéré [25] is used to predict the liquid film thickness and the wall film resistance.

It should be noted that the correlations of Leung et al. [1, 12] and Dai et al. [11] are developed based on the circular tubes in which the liquid film thickness is constant around the tube perimeter. However, the liquid film thicknesses are not uniform at the cross-section in square- and rectangularsection ducts as shown in Fig.6. Since no correlation has been developed to predict the average liquid film thickness in square and rectangular ducts, an average liquid film thickness at the center $(z=4 \mathrm{~mm})$ of the Taylor bubble, calculated by eq. (26), is used to calculate $N u_{f s}$ in the numerical work.

$$
\delta=\quad 1 /\left[1 / n \sum_{i=0}^{n}\left(1 / \delta_{i}\right)\right]
$$

where $\delta_{\mathrm{i}}$ is the liquid film thickness at a specific point $(i)$ on the liquid-gas interface, and $n$ is the total number of the points.

Fig. 11 shows the effect of $L_{s}{ }^{*}$ on $N u_{l}{ }^{*}$ and $N u_{f s}$. As expected, both $N u_{l}{ }^{*}$ and $N u_{f s}$ decrease with increasing $L_{s}{ }^{*}$, which means that the effect of the Taylor bubble on the heat transfer process in the liquid slug is more pronounced at higher $R e$ with a shorter liquid slug. $N u_{l}{ }^{*}$ for the EG/N $\mathrm{N}_{2}$ cases is lower than that for the $\mathrm{W} / \mathrm{N}_{2}$ cases at the same $L_{s}{ }^{*}$ because of the thicker liquid film. When the thermal resistance contribution of the liquid film is subtracted from the apparent slug Nusselt number, $N u_{f s}$

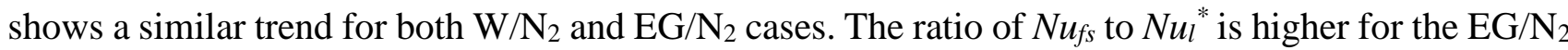
cases at the same $L_{s}{ }^{*}$, corresponding to the higher thermal resistance contribution of the wall film.

Based on the formulation of eq. (22) and (23), an attempt is made to develop correlations for predicting the heat transfer performance of gas-liquid Taylor flow in square- and rectangular-section ducts. The values of the constants in eq. (27) and (28) are determined by using least-squares fitting, and eq. (27) is validated for $L_{\mathrm{s}}^{*}>1.92 \times 10^{-4}$. The comparisons of the numerical data with the predictions are illustrated in Fig. 12. As shown in Fig. 12 (a) and (b), eq. (27) and (28) can predict the numerical data well, and nearly all the numerical data fall inside the $\pm 25 \%$ error bands. The MAD for eq. (27) 
and (28) is $14.2 \%$ and $10.4 \%$, respectively. Though these correlations are based on the numerical data, the reasonable predication obtained by eq. (27) and (28) confirms the applications of the basic formations in Leung et al. [1, 12] and Dai et al. [11] to Taylor flow in square- and rectangular-section ducts and provides a guide for future experimental investigations.

$$
\begin{gathered}
N u_{f s}=N u_{l o}+\frac{0.023 A R^{0.718}}{L_{s}^{*}-3.337 \times 10^{-3} L_{s}^{* 1 / 3}} \\
N u_{l}^{*}=N u_{l o}+0.0138 A R^{0.744} \mathrm{Ca}^{-0.255} L_{s}^{*-0.883}
\end{gathered}
$$

\section{Conclusions}

The thermal and flow characteristics of gas-liquid Taylor flow in vertical square- and rectangularsection ducts were studied numerically. The effects of fluid properties, flow parameters, and aspect ratios on the bubble shapes, friction factors, and Nusselt numbers were discussed. 26 cases, using water and ethylene glycol as the continuous phase with nitrogen as the disperse phase, were considered at two-phase mixing velocity $\left(v_{t p}\right)$ ranging from 0.089 to $0.446 \mathrm{~m} / \mathrm{s}$, corresponding to a wide range of Capillary number $(0.0011 \leq C a \leq 0.19)$. The simulations were conducted in a unit cell containing one Taylor bubble and two halves of the liquid slug in a frame of reference moving with the Taylor bubble. Two different methods were used to perform the reference frame moving: dynamic mesh method and frame motion method. The results obtained by these two methods are virtually identical, while the latter is numerically more efficient.

The Taylor bubble is confined by the duct wall in square- and rectangular-section ducts at lower $C a$, while an axisymmetric Taylor bubble is formed at higher $C a$. The bubble length increases with increasing $v_{t p}$ and $\mathrm{Ca}$ at a fixed gas void fraction, and the curvatures of the Taylor bubble tail and nose decrease with increasing $A R$. The liquid film thickness in the square and rectangular ducts is not uniform with a thicker liquid film formed at the tube corner. The recirculation volume decreases and the dimensionless recirculation time increases with increasing $C a$.

The friction factor decreases with increasing $v_{t p}$ and $A R$ and with decreasing $\varepsilon_{G}$, and the reverse is true for the two-phase Nusselt number. Compared with the EG/ $\mathrm{N}_{2}$ cases, the addition of a Taylor bubble plays a more significant role on the pressure drop increase and heat transfer enhancement for the $\mathrm{W} / \mathrm{N}_{2}$ cases because of the larger recirculation volume and smaller dimensionless recirculation 
time. The numerical pressure gradients fit well with the prediction of the modified Kreutzer et al. [10] correlation with a MAD of $6.3 \%$. Two correlations are developed to predict the apparent slug Nusselt number and the film-to-slug Nusselt number for gas-liquid Taylor flow in square and rectangular ducts. Though these correlations are based on the numerical data, the reasonable predication confirms the applications of the basic formulations in Leung et al. [1, 12] and Dai et al. [11] to Taylor flow in square and rectangular tubes and makes a guide for future experimental investigations.

\section{Acknowledgements}

This work is supported by National Science Foundation of China (51210011) and National Science Foundation of Zhejiang Province (LZ13E060001). 


\section{Reference}

[1] S. S. Leung, R. Gupta, D. F. Fletcher, B. S. Haynes, Effect of flow characteristics on Taylor flow heat transfer, Ind. Eng. Chem. Res. 51(4) (2011) 2010-2020.

[2] K. Triplett, S. Ghiaasiaan, S. Abdel-Khalik, D. Sadowski, Gas-liquid two-phase flow in microchannels Part I: two-phase flow patterns, Int. J. Multiphase Flow 25(3) (1999) 377-394.

[3] H. Liu, C. O. Vandu, R. Krishna, Hydrodynamics of Taylor flow in vertical capillaries: Flow regimes, bubble rise velocity, liquid slug length, and pressure drop, Ind. Eng. Chem. Res. 44(14) (2005) 4884-4897.

[4] C. Yao, Y. Zhao, C. Ye, M. Dang, Z. Dong, G. Chen, Characteristics of slug flow with inertial effects in a rectangular microchannel, Chem. Eng. Sci. 95(0) (2013) 246-256.

[5] Y. Han, N. Shikazono, Measurement of the liquid film thickness in micro tube slug flow, Int. J. Heat Fluid Flow 30(5) (2009) 842-853.

[6] Y. Han, N. Shikazono, Measurement of liquid film thickness in micro square channel, Int. J. Multiphase Flow 35(10) (2009) 896-903.

[7] A. C. Devoria, K. Mohseni, Droplets in an axisymmetric microtube: Effects of aspect ratio and fluid interfaces, Phys. Fluids 27(1) (2015) 012002-1-18.

[8] C. Meyer, M. Hoffmann, M. Schlüter, Micro-PIV analysis of gas-liquid Taylor flow in a vertical oriented square shaped fluidic channel, Int. J. Multiphase Flow 67(0) (2014) 140-148.

[9] M. J. F. Warnier, M. De Croon, E. V. Rebrov, J. C. Schouten, Pressure drop of gas-liquid Taylor flow in round micro-capillaries for low to intermediate Reynolds numbers, Microfluid. Nanofluid. 8(1) (2010) 33-45.

[10] M. T. Kreutzer, F. Kapteijn, J. A. Moulijn, C. R. Kleijn, J. J. Heiszwolf, Inertial and interfacial effects on pressure drop of Taylor flow in capillaries, AlChE J. 51(9) (2005) 2428-2440.

[11] Z. Dai, Z. Guo, D. F. Fletcher, B. S. Haynes, Taylor flow heat transfer in microchannels-Unification of liquidliquid and gas-liquid results, Chem. Eng. Sci. 138 (2015) 140-152.

[12] S. S. Y. Leung, Y. Liu, D. F. Fletcher, B. S. Haynes, Heat transfer in well-characterised Taylor flow, Chem. Eng. Sci. 65(24) (2010) 6379-6388.

[13] Y. S. Lim, S. C. M. Yu, N. T. Nguyen, Flow visualization and heat transfer characteristics of gas-liquid twophase flow in microtube under constant heat flux at wall, Int. J. Heat Mass Transfer 56(1-2) (2013) 350-359.

[14] P. A. Walsh, E. J. Walsh, Y. S. Muzychka, Heat transfer model for gas-liquid slug flows under constant flux, Int. J. Heat Mass Transfer 53(15-16) (2010) 3193-3201.

[15] P. Angeli, A. Gavriilidis, Hydrodynamics of Taylor flow in small channels: a review, Proceedings Of the Institution Of Mechanical Engineers Part C-Journal Of Mechanical Engineering Science 222(5) (2008) 737-751.

[16] R. Gupta, D. Fletcher, B. Haynes, Taylor flow in microchannels: a review of experimental and computational work, J. Comput. Multiph. Flows 2(1) (2010) 1-31.

[17] M. Mac Giolla Eain, V. Egan, J. Howard, P. Walsh, E. Walsh, J. Punch, Review and extension of pressure drop models applied to Taylor flow regimes, Int. J. Multiphase Flow 68(0) (2015) 1-9.

[18] T. Bandara, N. T. Nguyen, G. Rosengarten, Slug flow heat transfer without phase change in microchannels: A review, Chem. Eng. Sci. 126(0) (2015) 283-295.

[19] M. Akbar, S. Ghiaasiaan, Simulation of Taylor flow in capillaries based on the volume-of-fluid technique, Ind. Eng. Chem. Res. 45(15) (2006) 5396-5403.

[20] S. Laborie, C. Cabassud, L. Durand-Bourlier, J. M. Lainé, Characterisation of gas-liquid two-phase flow inside capillaries, Chem. Eng. Sci. 54(23) (1999) 5723-5735.

[21] D. Liu, S. Wang, Hydrodynamics of Taylor flow in noncircular capillaries, Chemical Engineering and Processing: Process Intensification 47(12) (2008) 2098-2106. 
[22] N. Shao, A. Gavriilidis, P. Angeli, Effect of Inlet Conditions on Taylor Bubble Length in Microchannels, Heat Transf. Eng. 32(13-14) (2011) 1117-1125.

[23] M. T. Kreutzer, M. G. Eijnden, F. Kapteijn, J. A. Moulijn, J.J . Heiszwolf, The pressure drop experiment to determine slug lengths in multiphase monoliths, Catalysis Today 105(3-4) (2005) 667-672.

[24] S. Irandoust, B. Andersson, Liquid-film in Taylor flow through a capillary, Ind. Eng. Chem. Res. 28(11) (1989) 1684-1688.

[25] P. Aussillous, D. Quéré, Quick deposition of a fluid on the wall of a tube, Phys. Fluids 12(10) (2000) $2367-2371$.

[26] D. M. Fries, F. Trachsel, P. R. Von Rohr, Segmented gas-liquid flow characterization in rectangular microchannels, Int. J. Multiphase Flow 34(12) (2008) 1108-1118.

[27] T. C. Thulasidas, M. A. Abraham, R. L. Cerro, Bubble-train flow in capillaries of circular and square crosssection, Chem. Eng. Sci. 50(2) (1995) 183-199.

[28] T. C. Thulasidas, M. A. Abraham, R. L. Cerro, Flow patterns in liquid slugs during bubble-train flow inside capillaries, Chem. Eng. Sci. 52(17) (1997) 2947-2962.

[29] R. S. Abiev, Modeling of pressure losses for the slug flow of a gas-liquid mixture in mini-and microchannels, Theor. Found. Chem. Eng. 45(2) (2011) 156-163.

[30] A. R. Betz, D. Attinger, Can segmented flow enhance heat transfer in microchannel heat sinks?, Int. J. Heat Mass Transfer 53(19-20) (2010) 3683-3691.

[31] M. T. Kreutzer, P. Du, J. J. Heiszwolf, F. Kapteijn, J. A. Moulijn, Mass transfer characteristics of three-phase monolith reactors, Chem. Eng. Sci. 56(21-22) (2001) 6015-6023.

[32] A. Mehdizadeh, S. A. Sherif, W. E. Lear, Numerical simulation of thermofluid characteristics of two-phase slug flow in microchannels, Int. J. Heat Mass Transfer 54(15-16) (2011) 3457-3465.

[33] R. Gupta, D. F. Fletcher, B. S. Haynes, CFD modelling of flow and heat transfer in the Taylor flow regime, Chem. Eng. Sci. 65(6) (2010) 2094-2107.

[34] R. Gupta, D. F. Fletcher, B. S. Haynes, On the CFD modelling of Taylor flow in microchannels, Chem. Eng. Sci. 64(12) (2009) 2941-2950.

[35] R. Gupta, S. S. Y. Leung, R. Manica, D. F. Fletcher, B. S. Haynes, Hydrodynamics of liquid-liquid Taylor flow in microchannels, Chem. Eng. Sci. 92(0) (2013) 180-189.

[36] A. N. Asadolahi, R. Gupta, S. S. Y. Leung, D. F. Fletcher, B. S. Haynes, Validation of a CFD model of Taylor flow hydrodynamics and heat transfer, Chem. Eng. Sci. 69(1) (2012) 541-552.

[37] J. D. P. Araújo, J. M. Miranda, A. M. F. R. Pinto, J. B. L. M. Campos, Wide-ranging survey on the laminar flow of individual Taylor bubbles rising through stagnant Newtonian liquids, Int. J. Multiphase Flow 43 (2012) 131-148.

[38] A. N. Asadolahi, R. Gupta, D. F. Fletcher, B. S. Haynes, CFD approaches for the simulation of hydrodynamics and heat transfer in Taylor flow, Chem. Eng. Sci. 66(22) (2011) 5575-5584.

[39] V. Talimi, Y. S. Muzychka, S. Kocabiyik, A review on numerical studies of slug flow hydrodynamics and heat transfer in microtubes and microchannels, Int. J. Multiphase Flow 39 (2012) 88-104.

[40] T. Taha, Z. Cui, CFD modelling of slug flow inside square capillaries, Chem. Eng. Sci. 61(2) (2006) 665-675.

[41] T. Taha, Z. Cui, Hydrodynamics of slug flow inside capillaries, Chem. Eng. Sci. 59(6) (2004) 1181-1190.

[42] C. J. Falconi, C. Lehrenfeld, H. Marschall, C. Meyer, R. Abiev, D. Bothe, A. Reusken, M. Schlüter, M. Wörner, Numerical and experimental analysis of local flow phenomena in laminar Taylor flow in a square mini-channel, Phys. Fluids 28(1) (2016) 012109

[43] T. Abadie, C. Xuereb, D. Legendre, J. Aubin, Mixing and recirculation characteristics of gas-liquid Taylor flow in microreactors, Chem. Eng. Res. Des. 91(11) (2013) 2225-2234.

[44] R. S. Abiev, Bubbles velocity, Taylor circulation rate and mass transfer model for slug flow in milli- and microchannels, Chem. Eng. J. 227 (2013) 66-79. 
[45] V. Talimi, Y. S. Muzychka, S. Kocabiyik, Slug flow heat transfer in square microchannels, Int. J. Heat Mass Transfer 62 (2013) 752-760.

[46] Z. Che, T. N. Wong, N. T. Nguyen, C. Yang, Three dimensional features of convective heat transfer in dropletbased microchannel heat sinks, Int. J. Heat Mass Transfer 86 (2015) 455-464.

[47] J. Brackbill, D. B. Kothe, C. Zemach, A continuum method for modeling surface tension, J. Comput. Phys. 100(2) (1992) 335-354.

[48] R. S. Abiev, Method for calculating the void fraction and relative length of bubbles under slug flow conditions in capillaries, Theor. Found. Chem. Eng. 44(1) (2010) 86-101.

[49] R. S. Abiev, Circulation and bypass modes of the slug flow of a gas-liquid mixture in capillaries, Theor. Found. Chem. Eng. 43(3) (2009) 298-306.

[50] W. B. Kolb, R. L. Cerro, Coating the inside of a capillary of square cross-section, Chem. Eng. Sci. 46(9) (1991) 2181-2195.

[51] M. T. Kreutzer, F. Kapteijn, J. A. Moulijn, J. J. Heiszwolf, Multiphase monolith reactors: Chemical reaction engineering of segmented flow in microchannels, Chem. Eng. Sci. 60(22) (2005) 5895-5916.

[52] A. L. Hazel, M. Heil, The steady propagation of a semi-infinite bubble into a tube of elliptical or rectangular cross-section, J. Fluid Mech. 470 (2002) 91-114.

[53] S. Kececi, M. Wörner, A. Onea, H.S. Soyhan, Recirculation time and liquid slug mass transfer in co-current upward and downward Taylor flow, Catalysis Today 147, Supplement (2009) S125-S131.

[54] M. Bahrami, M. M. Yovanovich, J. R. Culham, Pressure drop of fully-developed laminar flow in microchannels of arbitrary cross-section, J. Fluids Eng. 128(5) (2006) 1036-1044.

[55] R.K. Shah, K.L. London, Laminar Flow Forced Convection Heat Transfer and Flow Friction in Straight and Curved Ducts- A Summary of Analytical Solutions, M490-342, Stanford, California, November 1971. 

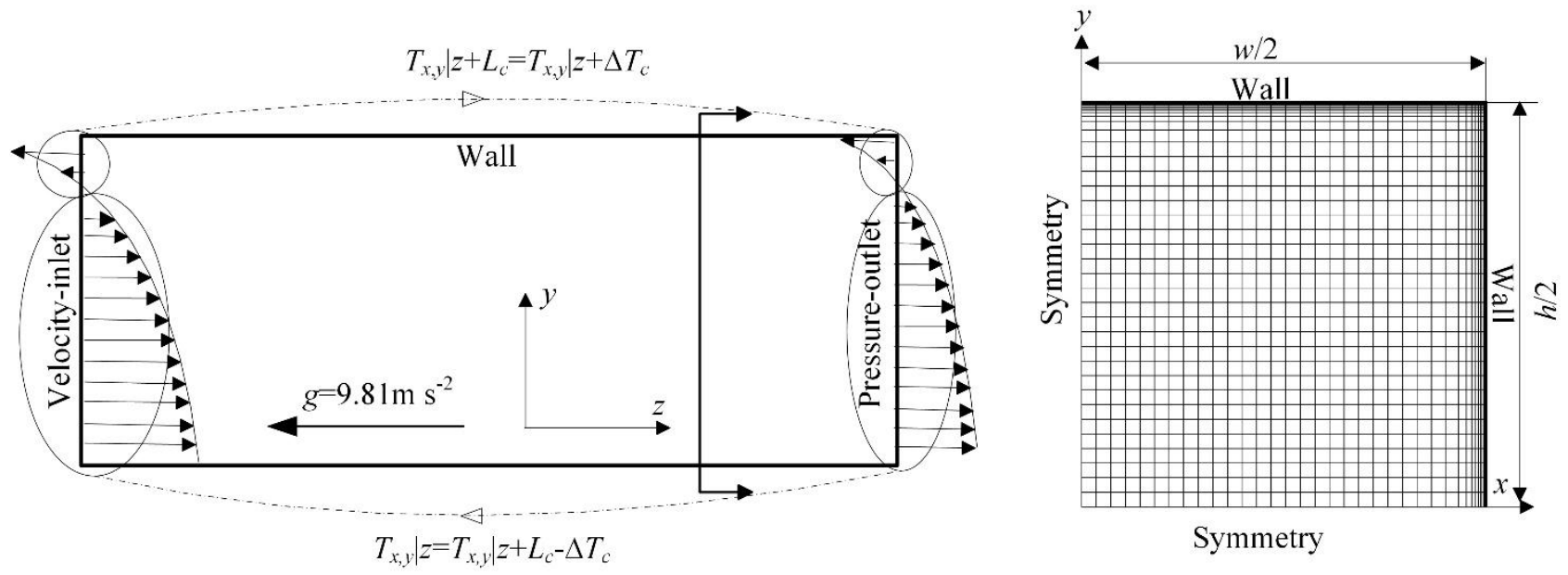

Fig. 1. Computational domain, boundary conditions, and sample mesh on a cross-section. The mesh size near the wall is refined to capture the thin liquid film of gas-liquid Taylor flow in square- and rectangular-section ducts. 


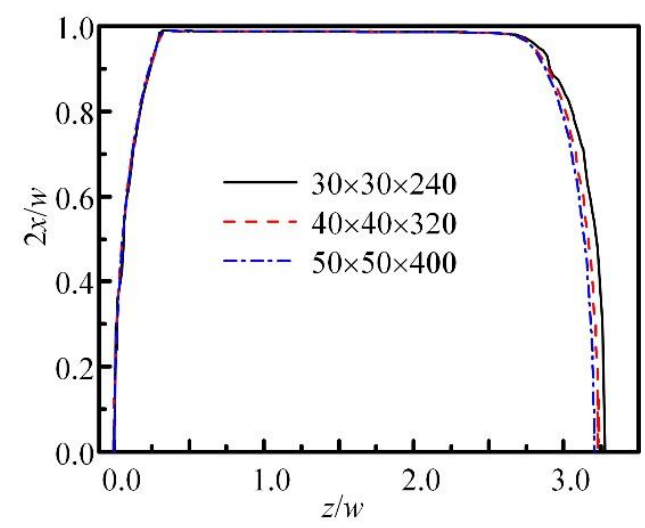

Fig. 2. Water/nitrogen Taylor bubble shapes on the $y=0$ plane obtained from three different mesh densities:30×30×240, 40×40×320, and 50×50×400. 


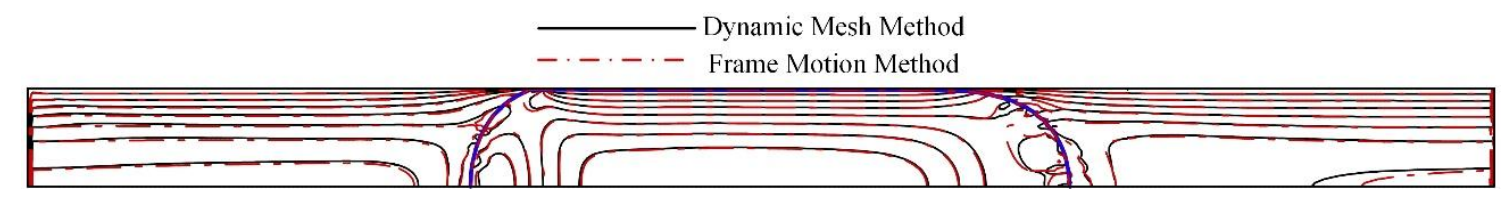

Fig. 3. Comparison of axial velocity contours for the dynamic mesh method (DMM) and frame motion method (FMM) methods. 


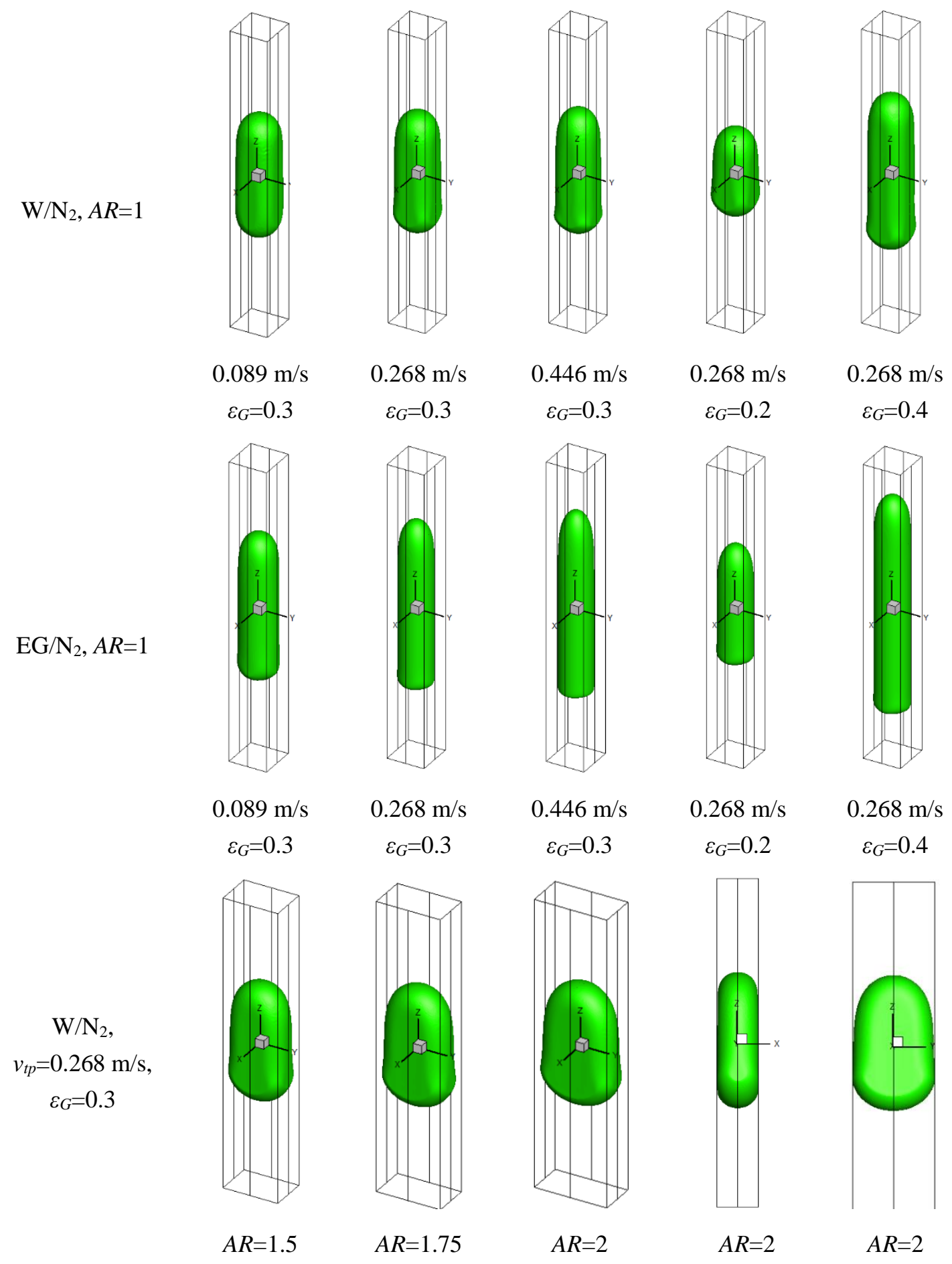




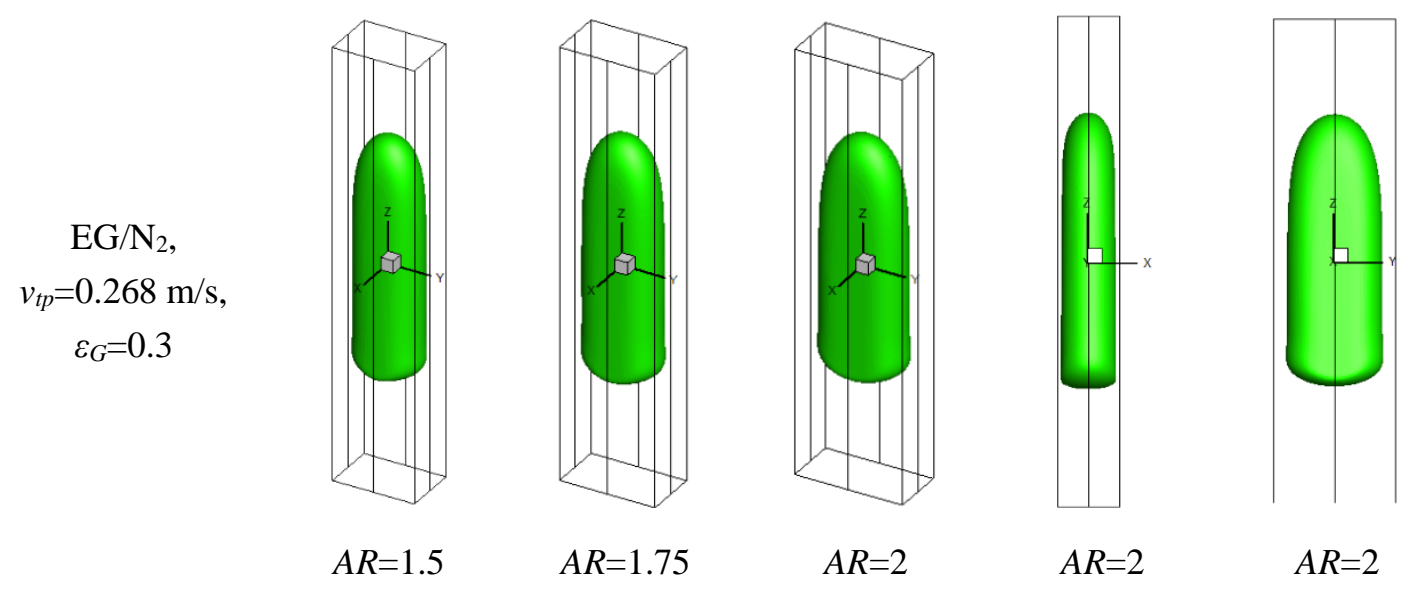

Fig. 4. Three-dimensional Taylor bubble shapes using water and ethylene glycol as the liquid phase at $v_{t p}$ ranging from 0.089 to $0.446 \mathrm{~m} / \mathrm{s}$ and $\varepsilon_{G}$ ranging from 0.2 to 0.4 in square- and rectangular-section ducts with $A R$ ranging from 1 to 2 . 


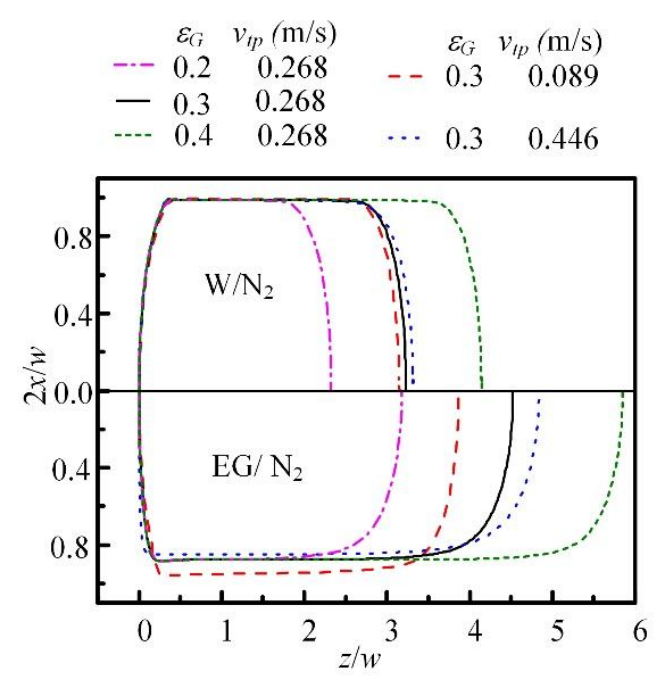

(a)

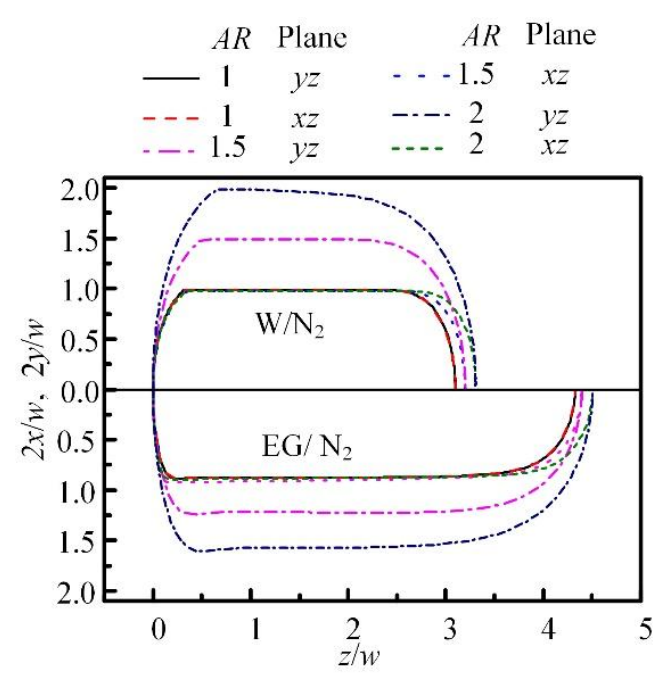

(b)

Fig. 5. Effects of (a) two-phase mixing velocity $(A R=1)$, gas void fraction $(A R=1)$, and (b) $A R\left(v_{\mathrm{tp}}=0.268\right.$ $\left.\mathrm{m} / \mathrm{s}, \varepsilon_{\mathrm{G}}=0.3\right)$ on Taylor bubble shapes for $\mathrm{W} / \mathrm{N}_{2}$ and $\mathrm{EG} / \mathrm{N}_{2}$ cases. 


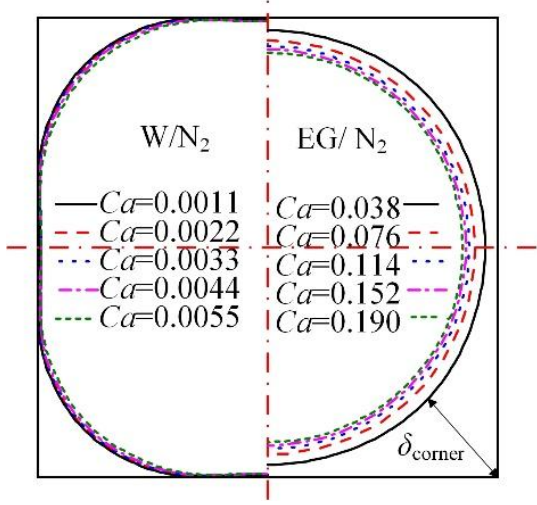

(a)

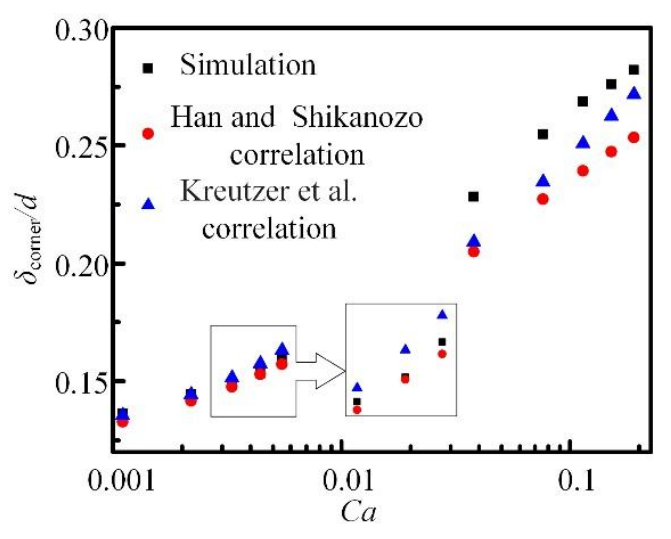

(b)

Fig. 6. (a) Evolution of the liquid-gas interfaces at a cross-section with increasing Capillary number (b) Comparison of numerical dimensionless liquid film thickness at the duct corners with correlations developed by Kreutzer et al. [51] and Han and Shikanozo [6]. 
(a)

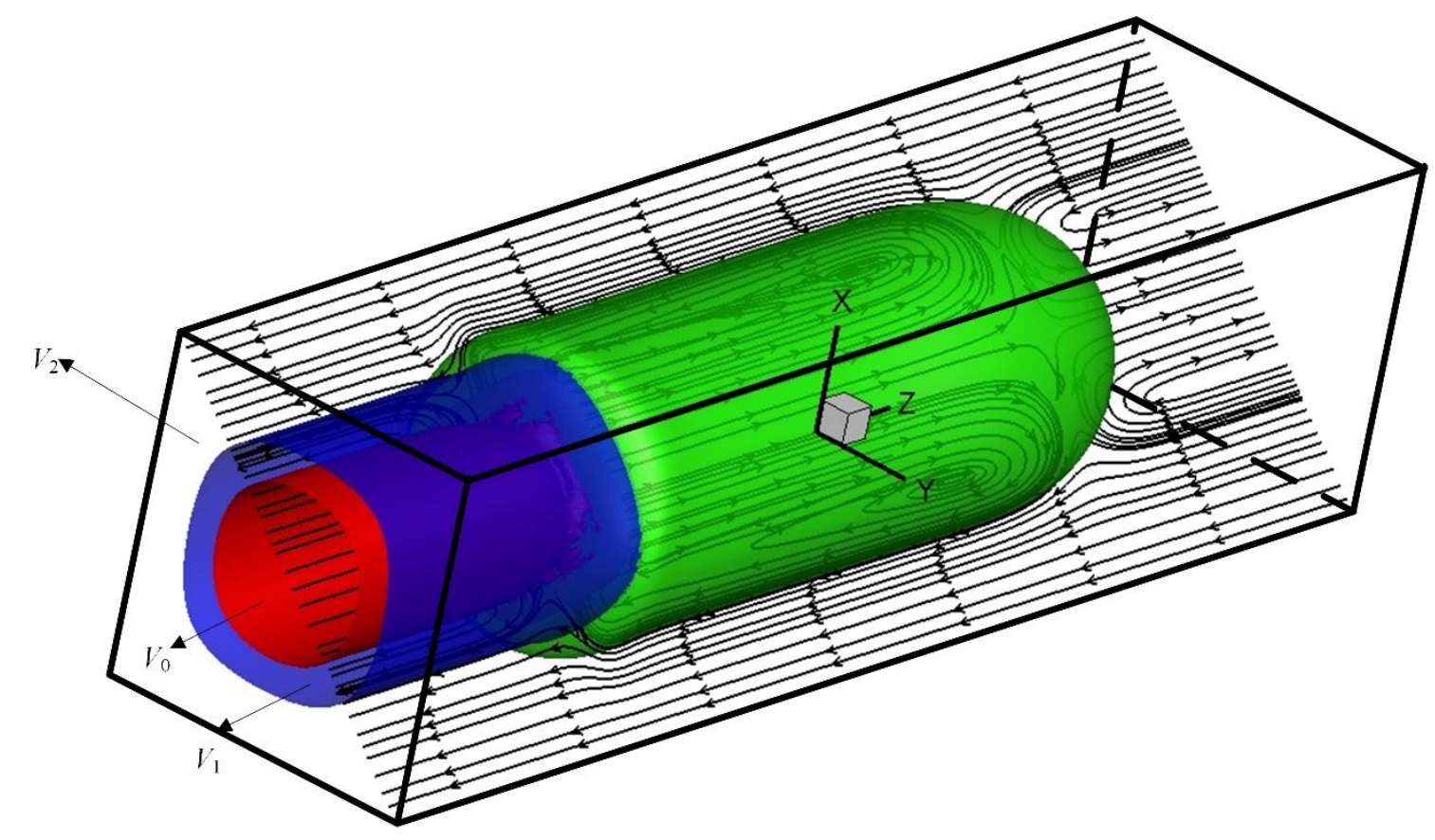

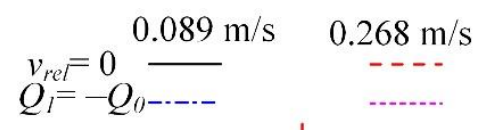

(b)

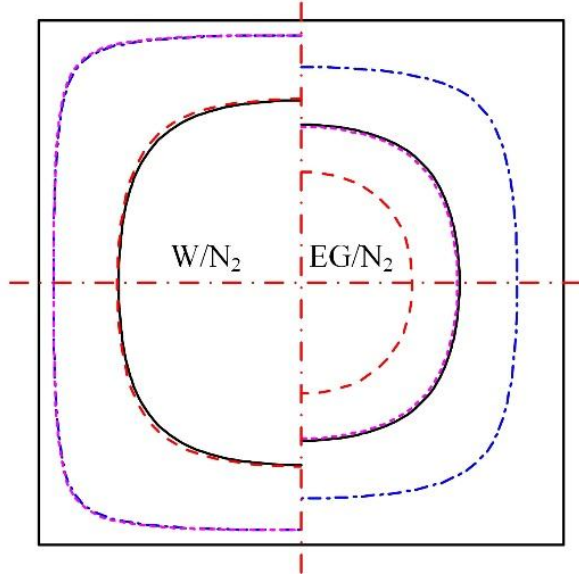

(c)

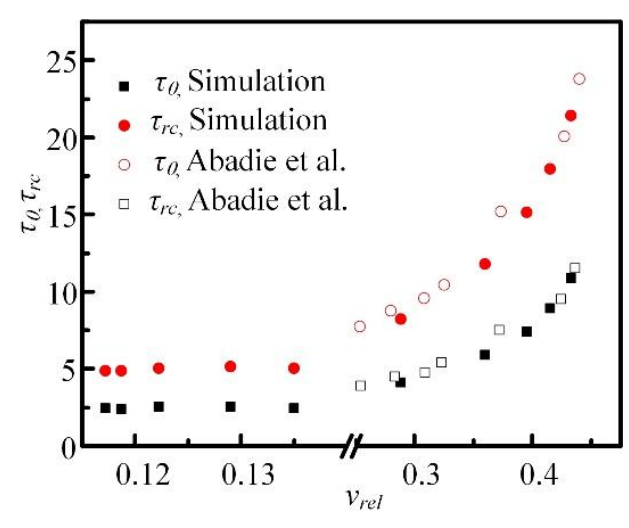

Fig. 7. (a) Schematic of flow on the diagonal plane together with the Taylor bubble and the recirculation regions. (b) Cross-sectional shape of the recirculation zones which is located within the transitional line $Q_{l}=-Q_{0}$ at $C a=$ 0.0011 and 0.0033 for $\mathrm{W} / \mathrm{N}_{2}$ cases and at $C a=0.0379$ and 0.1138 for EG/ $\mathrm{N}_{2}$ cases. (c) The effect of slip velocity on the dimensionless recirculation time. 


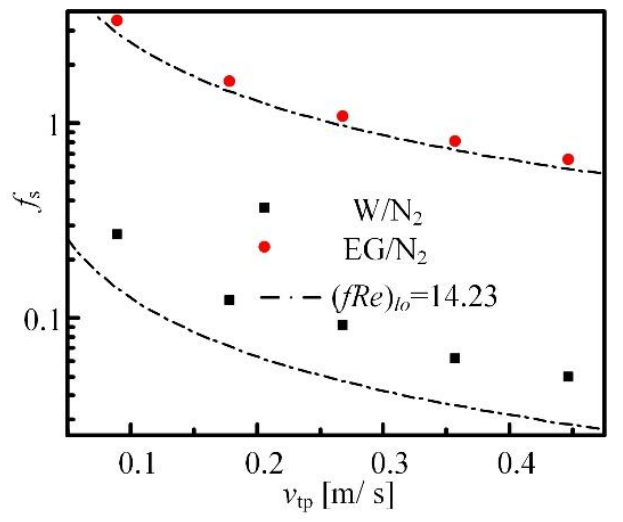

(a)

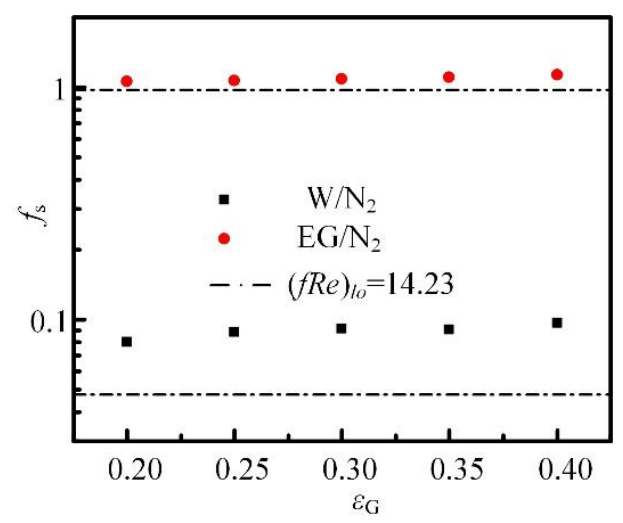

(b)

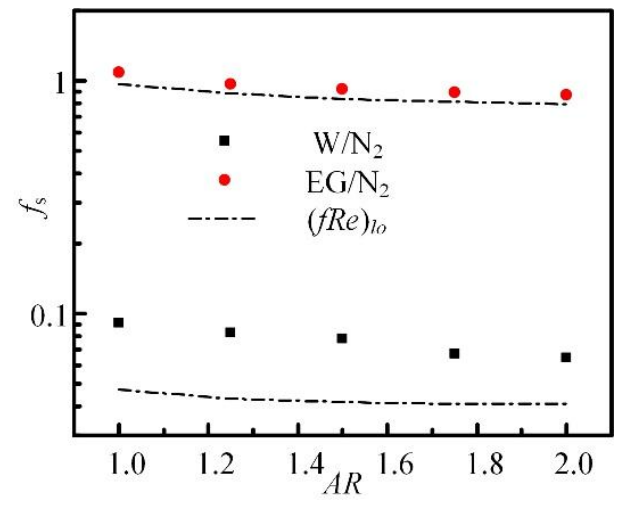

(c)

Fig. 8. Effects of (a) $v_{t p}$, (b) $\varepsilon_{G}$, and (c) $A R$ on the liquid slug friction factor $f_{s}$. 


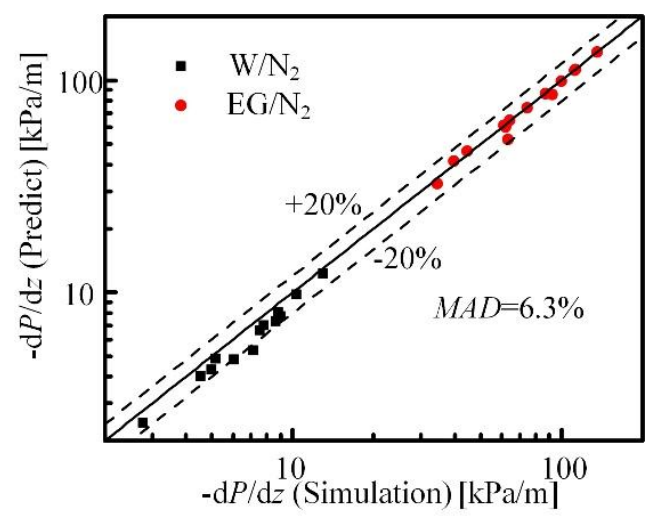

Fig. 9. Comparison of numerically simulated pressure gradients with predictions of Kreutzer et al. [10]. 


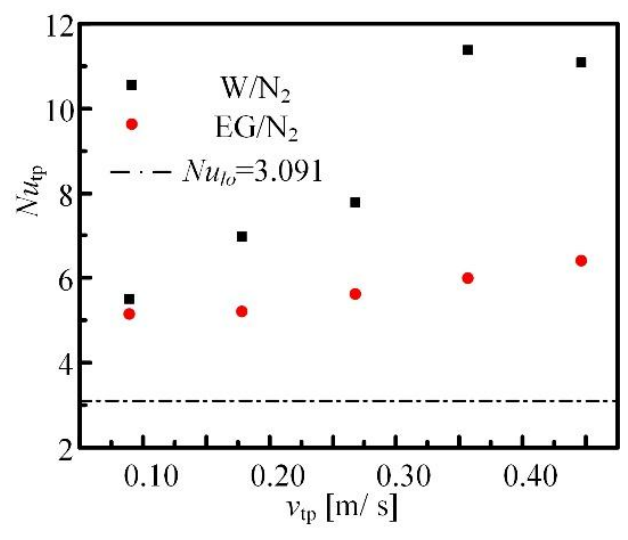

(a)

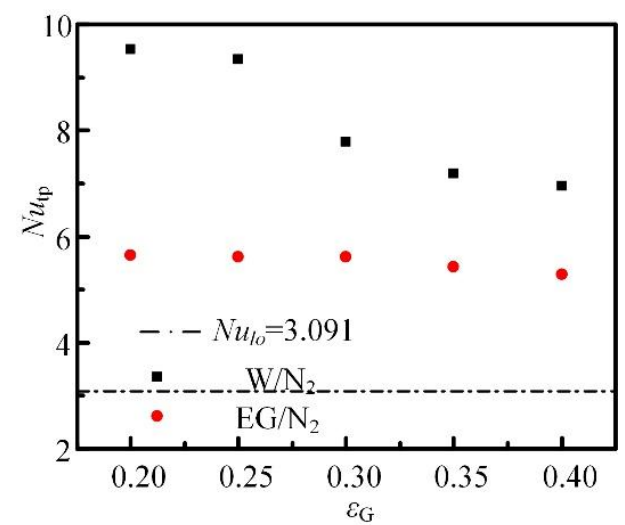

(b)

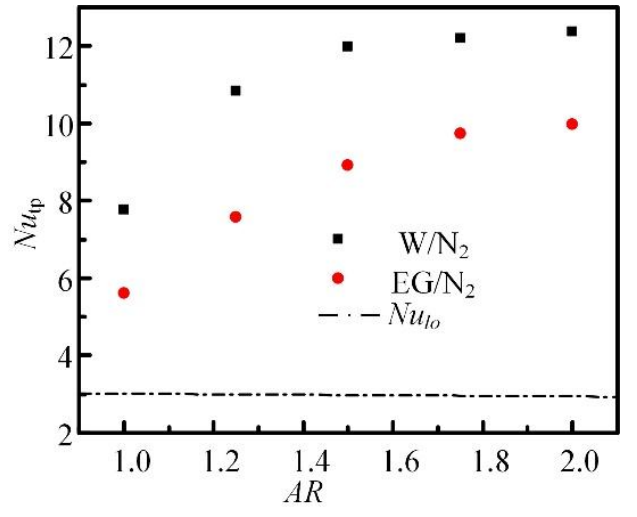

(c)

Fig. 10. Effects of (a) $v_{t p}$, (b) $\varepsilon_{G}$, and (c) $A R$ on the average Nusselt number $N u_{t p}$ 


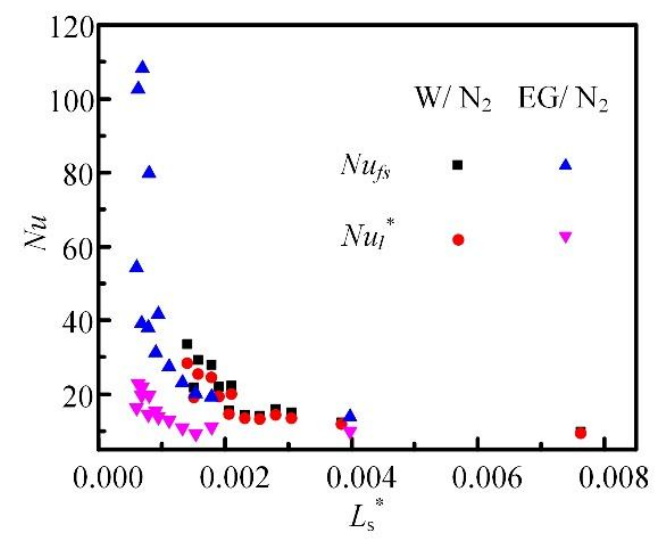

Fig. 11. The effect of normalized slug length $\left[L_{s}^{*}=L_{s} /\left(\operatorname{Re}_{t p} \operatorname{Pr}_{L} d\right)\right]$ on the apparent Nusselt number $N u_{l}{ }^{*}$ and the film-to-slug Nusselt number $N u_{f s}$. 


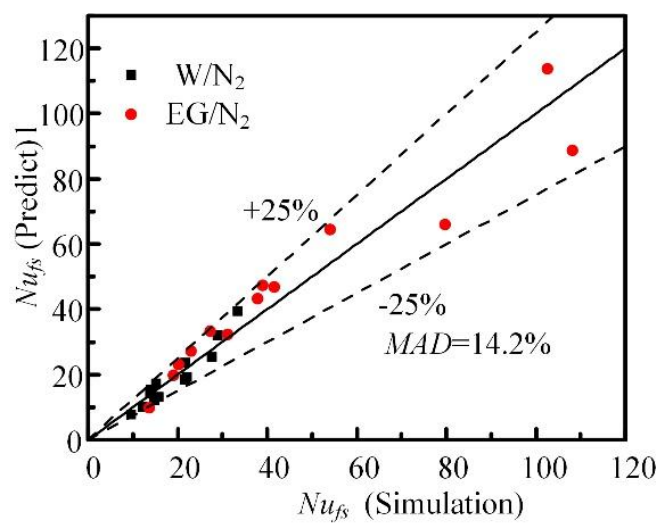

(a)

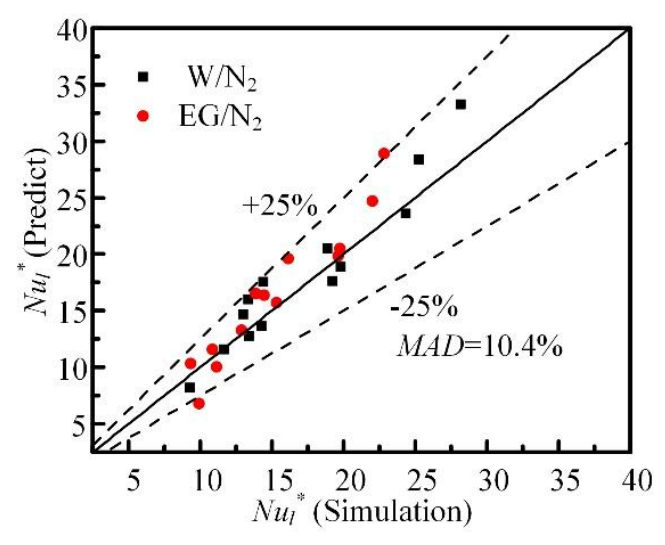

(b)

Fig. 12. Evaluation of the developed correlations for (a) the film-to-slug Nusselt number $N u_{f s}$ and (b) the apparent Nusselt number $\mathrm{Nu}_{l}{ }^{*}$ against the numerical data. 
Table 1.

Properties of working fluids

\begin{tabular}{cccc}
\hline Fluid & Nitrogen $\left(\mathrm{N}_{2}\right)$ & Water $(\mathrm{W})$ & Ethylene Glycol (EG) \\
\hline Density, $\rho\left(\mathrm{kg} / \mathrm{m}^{3}\right)$ & 1.145 & 997 & 1114 \\
Viscosity, $\mu(\mathrm{Pa} \cdot \mathrm{s})$ & $2.08 \times 10^{-5}$ & $8.90 \times 10^{-4}$ & 0.0204 \\
Specific heat capacity, $c_{p}(\mathrm{~J} / \mathrm{kg} \cdot \mathrm{k})$ & 1040 & 4182 & 2627 \\
Thermal conductivity, $k(\mathrm{~W} / \mathrm{m} \cdot \mathrm{K})$ & 0.0242 & 0.6 & 0.251 \\
Surface tension, $\sigma(\mathrm{N} / \mathrm{m})$ & & $\mathrm{W}^{\prime} \mathrm{N}_{2}=0.072$ & $\mathrm{EG} / \mathrm{N}_{2}=0.048$ \\
\hline
\end{tabular}


Table 2.

Parameters used in the cases studied

\begin{tabular}{cccccc}
\hline Working fluid & $A R$ & $v_{t p}(\mathrm{~m} / \mathrm{s})$ & $R e$ & $C a$ & $\varepsilon_{G}$ \\
\hline $\mathrm{W} / \mathrm{N}_{2}$ & 1 & $0.089-0.446$ & $100-500$ & $0.0011 \sim 0.0055$ & $0.2-0.4$ \\
$\mathrm{EG} / \mathrm{N}_{2}$ & 1 & $0.089-0.446$ & $4.87-24.37$ & $0.038-0.190$ & $0.2-0.4$ \\
$\mathrm{~W} / \mathrm{N}_{2}$ & $1.25-2$ & 0.268 & $333-400$ & 0.0033 & 0.3 \\
$\mathrm{EG} / \mathrm{N}_{2}$ & $1.25-2$ & 0.268 & $16.25-19.50$ & 0.114 & 0.3 \\
\hline
\end{tabular}

